\title{
Data Comics for Reporting Controlled User Studies in Human-Computer Interaction
}

\author{
Zezhong Wang, Jacob Ritchie*, Jingtao Zhou*, Fanny Chevalier, Benjamin Bach
}

\begin{abstract}
Inspired by data comics, this paper introduces a novel format for reporting controlled studies in the domain of humancomputer interaction $(\mathrm{HCl})$. While many studies in $\mathrm{HCl}$ follow similar steps in explaining hypotheses, laying out a study design, and reporting results, many of these decisions are buried in blocks of dense scientific text. We propose leveraging data comics as study reports to provide an open and glanceable view of studies by tightly integrating text and images, illustrating design decisions and key insights visually, resulting in visual narratives that can be compelling to non-scientists and researchers alike. Use cases of data comics study reports range from illustrations for non-scientific audiences to graphical abstracts, study summaries, technical talks, textbooks, teaching, blogs, supplementary submission material, and inclusion in scientific articles. This paper provides examples of data comics study reports alongside a graphical repertoire of examples, embedded in a framework of guidelines for creating comics reports which was iterated upon and evaluated through a series of collaborative design sessions.
\end{abstract}

Index Terms-Statistical communication, comics, scientific reports

\section{INTRODUCTION}

Effective communication in academic research is crucial, as it allows readers to assess the rigor of scientific methods and to build trust in scientific results. In particular, controlled experiments require detailed descriptions of key information related to procedures, hypotheses, conditions, data, and experimental setups, as well as careful reporting of data analysis and discussion of the results. Accessible presentation of this information is key not only for peer-review and replication, but for scientific dissemination, the training of students, and communication of research methodology and results to the general public. However, despite guidelines and conventions for technical writing, study reports often result in compressed descriptions that use domain-specific jargon and complex visualizations; important information and methodological decisions risk being buried, complex relations may not be sufficiently explained, or readers might simply overlook information that is crucial for interpretation, discussion, and application of the results [21], adding to research debt [60] and creating a need for interpretive labour [38].

To make research findings more accessible to both experts and nonexperts, a range of formats have emerged to complement traditional journal and conference articles. Examples include graphical abstracts [44], pictorials, posters, videos, interactive explanations [75], and academic blogs $[3,5]$ which have become one of the key factors initiating and increasing public engagement and effective communication [31,47]. These formats, often created by professionals [44], make compelling use of graphical representations, animation, interaction, and provide more space for detailed and understandable explanations, appropriate for a specific audience. However, this often comes at the cost of over-simplification, which can cause important details about methods, conditions, decisions, and context to be sacrificed in order to create compelling narration and content that is more easily understood.

In seeking to integrate and allow for both detailed presentation of studies and easier access by readers-experts and non-experts - this

- Zezhong Wang and Benjamin Bach are with The University of Edinburgh. E-mail: \{zezhong.wang,bbach\}@ed.ac.uk.

- Jacob Ritchie is with Stanford University.E-mail: jritchie@ stanford.edu.

- Jingtao Zhou is with The University of Edinburgh and Tianjin University. E-mail: i@tzingtao.com

- Fanny Chevalier is with University of Toronto.

E-mail: fanny@cs.toronto.edu.

*Jacob Ritchie and Jingtao Zhou contributed equally to the project.

Manuscript received xx xxx. 201x; accepted xx xxx. 201x. Date of Publication xx xxx. 201x; date of current version xx xxx. 201x. For information on obtaining reprints of this article, please send e-mail to: reprints@ieee.org. Digital Object Identifier: $x x . x x x x / T V C G .201 x . x x x x x x x$ paper explores the use of data comics $[11,84]$ to report controlled user studies in Human-Computer Interaction (HCI) and visualization. Our goal is to provide a framework for researchers and science communicators, guiding the dissemination process and designing visual narratives for scientific study reports (Figure 1). We opted for comics because of their great flexibility and numerous characteristics beneficial to communication, including $(i)$ tight integration of visual and textual explanations, (ii) sequential presentation of information, (iii) visual content for recall and quick navigation [79], and (iv) different levels of reading, i.e., quick overview as well as access to details, from skimming to close reading. As a static medium, comics are ideal for storyboarding and ideation [77]; there are minimal technical barriers to production, and they can be shared in many forms such as scientific papers, conference posters, slide shows, grant proposals and blogs. Moreover, comics are different from cartoons, as the basic principles of comics can fit different visual styles to make communication appropriate for a diverse set of audiences and media, e.g., ranging from hand sketches over vector graphics to computer generated visualizations $[10,14,50]$.

Based on five design objectives we explore the use of comics and data comics design patterns [13] to explain study setups and data analysis for controlled experiments, through a guided design discussion and a series of design iterations focused on previously published studies (Section 3). We then elaborate a 10-stage framework to guide the storyboarding of data comics for controlled user studies (Section 4), including stages such as context, hypotheses, data transformation, result presentation, etc. For each stage, we describe what information should be shown and discuss design solutions that we found in our exploration and working with seven participants in remote collaborative design sessions (Section 5). The collaborative design process and guidelines engaged participants in storyboarding comics for their own studies. We report on visual solutions for common tasks such as sketching hypotheses, reporting randomization, and detailed analysis of results. Feedback from participants highlights the need for improved reporting methods as well as the potential of data comics.

We do not aim to standardize reporting or to provide a visual grammar for using data comics. Nor do we intend to replace other forms of reporting studies and science. Rather, through our framework, which describes critical information that should be retained when simplifying study reporting for visual presentation, we hope to provide guidance and best practices to encourage and inspire researchers and science communicators to engage in both rigorous reporting and efficient explanation of their studies at different levels of detail according to the respective audience. Application scenarios include scientific and public talks, scientific posters, teaching materials and text books. Beyond HCI and visualization, our approach can generalize to other domains, given additional practice and exploration. A comic gallery, workshop material, and stage descriptions can be found at https://statscomics.github.io. 


\section{BACKGROUND}

\subsection{Scientific Reporting}

To support writers, reviewers, and readers, numerous international groups have published guidelines to standardize the information that should be provided in publications on the design, conduct, and analysis of the experiments [4]. Guidelines exist for clinical trials [68], animal research [49], observational studies in epidemiology [72], and communication of empirical results in HCI [32] -including checklists of information to include when reporting a randomised trial [40] or in-vivo experiment [49]. Some guides prescribe estimation approaches over dichotomous testing procedures for increased interpretability [32]. Other guidelines are established more implicitly through community norms, examples in highly-read papers, and good scholarly training.

Still, technical reports may not always result in a clear and comprehensive account of the research [59]. Moreover, as publication migrates online, it has become increasingly easy to append supplemental materials to research articles. Open data repositories, project websites, and data analysis scripts are now commonly used as a transparent demonstration of materials and study procedures to promote replication [42]. While this is extremely valuable, it adds to the complexity of information, and given the increasing pace of publication in many fields, even trained scientists can experience problems with understanding reported information and interpreting the output of statistical tools. Misconceptions and fallacies [32] as well as an increasing demand for the piecing together of information from multiple sources can lead to misguided or incorrect conclusions [18,51], and well-documented problems such as publication bias and the file drawer effect inflate the number of incorrect claims in the literature $[17,64,66]$.

In our work, we go beyond the traditional article format, exploring ways to report experiments - in line with scientific standards - that promote access to scientific knowledge while preserving transparency.

\subsection{Presentation and Explanation}

In addition to prose, graphical content is key to reporting scientific methods and results [55,61]. Figures in the form of graphs, diagrams, pictures, illustrations, and other data visualizations show experimental procedures [48], study setups [46], theoretical models [16], and research hypotheses $[35,41]$. Besides diagrams and illustrations, data visualizations are a key means of conveying results graphically by showing mean values, distributions, confidence intervals, outliers and other sources of uncertainty. Symbolic representations, e.g., for confidence intervals or quartiles in boxplots, can provide hurdles for novice readers [30] and should be explained [77].

There have been a number of suggestions to change or augment scientific reporting for better explanation. For example, Document Cards [71] provide for automatic summaries of scientific papers, including keywords and figures to facilitate browsing large document collections for relevance. However, our focus is not on concise summaries but on understandable explanations. In this vein, fluid documents $[82,83]$ allow readers to pull up supplemental information in-context, whereas elastic documents [15] support linking of textual and tabular content to automatically generated visualizations. Other new formats harness the ability of papers in HTML and PDF to include animation and interactivity to convey dynamic behaviours in user interfaces [39] or let readers explore variability in result outcomes across multiple analysis settings [33]. Similarly, the need to understand complicated concepts in machine learning and data science paired with an increasing (public, scientific, juristic, etc.) demand for transparency has led to an increase in interactive explorable explanations. Pioneered by Bret Victor $[75,76]$ and now widely disseminated by platforms such as Distill.pub [1], explorable explanations leverage interactive visualizations to explain complex concepts [6]. In general, while there exist many other sources to explain scientific methods (t-tests, ANOVA, etc.) our work focuses on the analysis pipeline-its stages and the decisions made for the conditions, factors, and setup, as well as the reporting of results-rather than explaining the functioning of a specific aspect of, e.g., a statistical model.
Our work is closer to graphical abstracts, a particular and increasingly common format to illustrate the core idea of a research paper through a single and concise graphic that can shows concepts, research methods, experimental setups, and results [44]. Comics, in comparison, provide for more space and narrative elements to deal with complex information and to enable a more in-depth understanding of a study's protocol and results.

\subsection{Comics for Explaining Science and Data}

Comics provide a unique medium for the communication of complex content. Their expressive and compelling power for visual storytelling comes from their unique combination of text and pictures $[29,36,56]$ and its linear reading order, able to break down complexity into sequential steps that a reader can consume at their own pace. By providing several panels on the same page, comics can also support overview and detail, allowing for simultaneous explanation and exploration. Empirical studies have shown the effectiveness of comics for presenting scientific phenomena [36] and data visualizations [77].

In a scientific and educational context, comics have been valued for their ability to promote public engagement [58], e.g., in stem cell research [8], nanotechnology [53], and many other domains [36]. Comics have also been used for classroom instruction of STEM topics, showing promising increases in student engagement $[43,70]$. Comics have been used to teach concepts in statistics $[37,62,74]$ such as means, medians, and regression, as well as concepts in data visualization [78].

The approach we present in this paper differs from such uses of comics to teach statistics or visualization, since the focus is not on educating or explaining particular statistical methods, but on reporting how such methods are used in a specific scientific analysis, to allow a reader to understand the purpose they serve. This is closer to the concept of a data comic [11,84], a genre that focuses on effective narration and explanation with data visualizations. Data comics offer a large design space comprising many data comic design patterns [13] and explored in previous workshops [77].

Thus, rather than understanding comics as sketchy 'cartoons' or as just an educational resource for public outreach, we explore comics as an effective means of communicating key information and decisions for reporting controlled, quantitative empirical studies in human computer interaction (HCI) and visualization. There is a low barrier to creating publication-ready comics, as well as universal shareability through paper publications, conference posters, textbooks, slideshows and websites. To the best of our knowledge, no examples, structured design explorations, or studies exist on the topic of using comics to report on scientific studies.

\section{DATA COMICS FOR REPORTING STUDIES}

\subsection{Design Objectives}

In exploring data comics for controlled user studies in HCI and visualization, we aim to investigate challenges and design solutions for the following design objectives O1-O5. Each objective marks an individual aspect of our approach to addressing study reporting.

01: Clarify decisions and important information: We aim to clarify key steps and decisions across methods, protocols, and results. This includes information about study setup, number and demographics of participants, conditions and datasets used in trials, user interfaces, or data transformations such as outlier removal. Each of these decisions has implications for interpreting the results. Our goal is to present and provide for visual explanation $(\mathrm{O} 2)$ of these decisions to facilitate the interpretation of results.

O2: Explain information visually: Our exploration focuses on effective visual explanation of the information in a study report. Where appropriate, we want to find visual depictions to explain hypotheses, data samples, study protocols, task conditions and data transformations and to explain results through data visualization. The repertoire of visual depictions may range from diagrams and schemata, to graphic pictograms and annotations, screenshots, illustrations, and data visualizations. Textual or other narrative structures present in comics are outside our scope. 
O3: Support recognition and recall with a visual vocabulary: $\mathrm{Be}-$ sides using visual explanations $(\mathrm{O} 2)$ for understanding key information $(\mathrm{O} 1)$ and providing for overview (O4), visual information can afford quick reference through recall and recognition. For example, an effect discussed in the results section of a study report may be present only for a specific condition, which can be reviewed by glancing back to the relevant section of the comic. A visual vocabulary made up of recognizable symbols for each condition might facilitate this process. While earlier work has found benefits of data comics for memorizing content [79], investigating memorability of comics is beyond this paper's scope.

04: Provide visual overview and structure to facilitate information retrieval: For example, individual stages of a study or analysis could inform the glanceable higher level structure of the comic. At the same time, we aim to provide space for sufficiently detailed, specific information, e.g., though dedicated panels of different sizes. Reading the comic at different levels of detail could help make comics-style study reports compelling to different audiences, including both experts and non-experts.

05: Use aesthetic appeal to create engagement: Finally, besides the rather functional objectives $\mathrm{O} 1-\mathrm{O} 4$, we posit that reporting studies through comics can provide for a fresh view of science through compelling visual narratives and clear visual styles (e.g., hand-drawn illustrations and characters). We believe comicsprovided the right visual style is used-can engage non-experts and larger audiences, further promoting science outreach.

\subsection{Methodology}

Our methodology was driven by the need to create comics for studies, due to the lack of existing examples. Following a method used in prior research $[10,13]$, we began by creating comics for studies reported in existing academic papers and eventually created 15 comic sketches for 13 papers, 4 of which we polished and which can be found on our website. Our detailed methodology was as follows.

1. Design Exploration: We started our exploration by creating comics of our own papers that involved empirical studies, since we were most familiar with the intricate details of these experiments: $[7,12,26,63,79]$. As our research lies within visualization and HCI, our papers inherently contained a number of helpful visual resources that were used as the basis of the visual vocabulary. We constructed at least one complete example comic for each study, starting with sketching on paper, and later translating to sketching on drawing boards, which allowed for quick iteration and exploration of ideas, layouts, and visual styles. During this iterative process which involved discussions among all authors, we were aided by data comic design patterns [13] and existing guidelines for visually explaining data visualization techniques [78].

2. Design Discussion: In parallel to our own exploration, we conducted one 1.5 hour design discussion with three participants (two graduate students specializing in HCI and one undergraduate), who were asked to create data comics for a research paper that they were familiar with, with an objective of using visual explanations to support understanding of the analysis. The discussion was introduced using a presentation, during which participants were shown examples of data comics, facilitated by one of the authors. Participants first created written lists of all steps of the analysis, which they then turned into comic sketches with pen and paper.

Resulting data comics showed integrated use of visual and textual explanation, with a clear reading order indicated by comic panels (details are discussed in Sect. 6). These comics provide visual solutions for illustrating data collection, using annotated illustrations (e.g., interaction between a user and a smart watch), task procedures described using a simple flowchart, and interpretations of study results (e.g., a bar chart showing an overall trend, with annotated details beside one of the bars). All three participants included narrator characters to explain study details. In the discussion following the workshop, participants commented that data comics could reveal concrete information such as the effects of outlier removal or enable critical reasoning about whether 'outliers' were really outliers.
However, participants reported that they struggled to decide which parts of the analysis should be included into the comic. One reason mentioned was the lack of familiarity with studies: "my level of comfort with stats is too low to do the translation to comic manually". Such comments motivated us to identify information to be included before conducting any further evaluation (Sect. 4).

3. Design by stages and examples: To promote transparency and the reporting of key information, we consulted existing checklists and guidelines for scientific reporting (Sect. 2.1). We analysed examples produced during our exploration and design discussion, and distilled an initial list of stages and design solutions reported in Sect. 4. To evaluate whether our stages could effectively serve as a prescriptive guide to creating comic-style study reports, we asked a computer science student with design skills who was unaware of our research to create more data comics (he now is a co-author of this paper). The generated examples clearly presented the structure of the stages and effectively applied design solutions.

To further explore the usefulness of data comics in presenting research, we extended our practice to HCI studies about music [69] and speech language processing [65]. The inclusion of a particular paper was based on two principles: $i$ ) it included a controlled user study, and ii) we were familiar with the topic so that we could maximize our effectiveness in explaining the hypotheses, procedures and analyses. During this process we kept developing and refining our list of stages and associated design solutions (Sect. 4).

4. Collaborative design: Eventually, to further evaluate our stages and the idea of using data comics for reporting, to source more design solutions, as well as to find problems and/or solutions not yet covered by our own explorations, we conducted another set of collaborative design sessions with seven researchers (five doctoral and two post-graduate) (Sect. 5).

\subsection{Example Comic: Weighted Graph Comparison}

To better explain what information our comics aim to encompass, and how this information is visually presented with respect to our design objectives O1-O5 (Sect. 3.1), this subsection discusses an example data comic for a published study. Fig. 1 shows a comic illustrating a study on comparing weighted networks as conducted by Alper et al. [7]. The paper presents a classic example of a controlled user study in visualization; in a lab setting, it compares two visualization techniques and tests four hypotheses on task accuracy and task-completion time. The comic is meant for a blog, project website, or conference poster.

Structure: The comic in Fig. 1 is subdivided into four parts I-IV by the author to highlight important stages of the study report (O1). Panel numbers 1-36 are defined in the bottom left corner for each panel of the comic. The comic begins (I) by introducing context and study motivation, including key concepts (e.g., weighted graphs in panels 1-4), purpose of the study and research question 4. It also introduces the subject of study, i.e., the six visualizations of interest 6 . Both concepts and conditions (I) are introduced with illustrations which are reused throughout the remainder of the comic, providing visual consistency and quick visual reference. Then (II), the comic introduces tasks and conditions examined in the study. It does so by introducing formal notions for the two conditions and visual explanations for each condition 9-10, using the anatomy cheat sheet described by Wang et al. [78]. Each task 12-14 is explained through a vertical sequence of images explaining the task's goal, what participants had to look for, how they entered their answers, and the set of possible answers. The last panel in part II introduces the different data sets and which parameters were used to generate or select them.

Part III of this comic describes each of the four hypotheses $17-20$ for the study by repeating visual depictions of relevant conditions involved in each hypothesis (task, datasets). In addition, each hypothesis illustrates the expected results in terms of the dependent variable, if that hypothesis should hold, using annotated bar chart sketches. Part IV details the study setup, including participants, setup, conditions, and a reconstructed screenshot of the interface presented to participants 2122. Panels 25-28 show that the order in which data sets were shown 
I

\section{Context, Motivation} $\&$ Problem Study

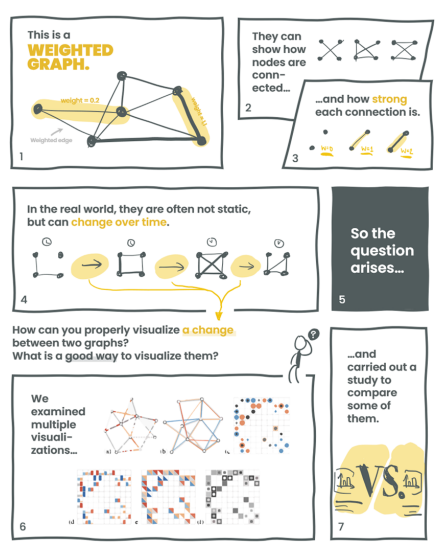

II

Tasks \&

Conditions

-

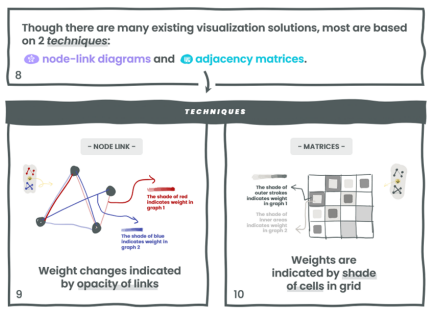

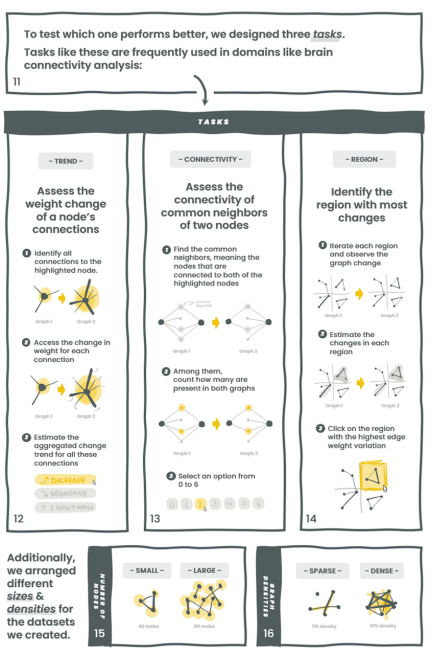

III

Hypotheses
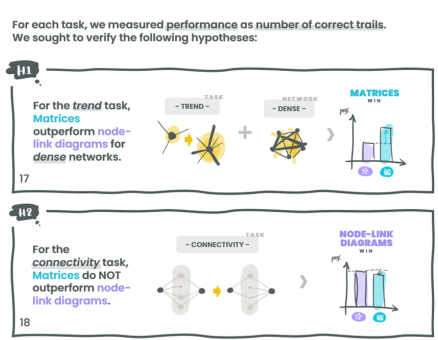
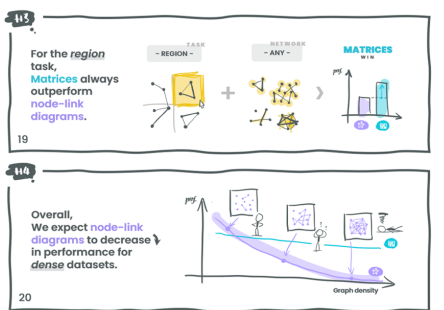

IV

Study Setup

Data Collection \&

Data Transformation

-

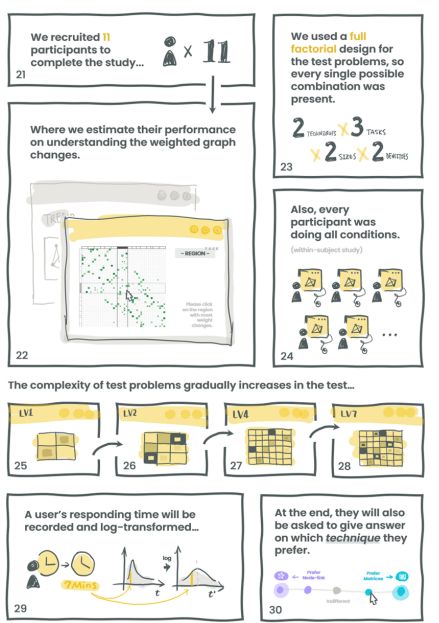

V

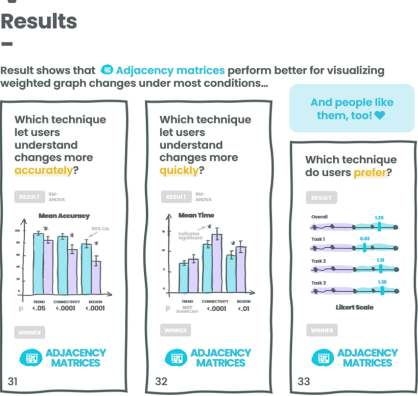

VI

Hypotheses Evaluation

2म4
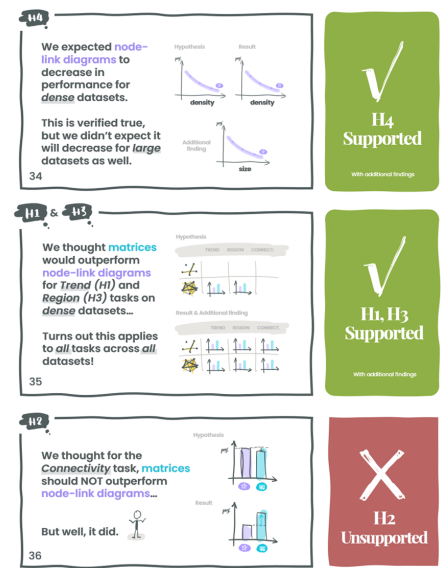

Fig. 1. Example of a data comic illustrating a study that compared two visualization techniques for weighted networks. Created by one of the authors through an iterative process, based on information provided in the original paper [7]. Each panel is labeled with a number in the bottom left corner, which we reference in (Sect. 3.3).

goes from simple to difficult. The last two panels in IV illustrate that task completion time has been log-transformed 29 and that participants were asked for a subjective ranking on a Likert scale 30 .

The results section of the comic (V) displays results for each of the independent variables, highlights significant findings, and 'declares' the winner technique for independent variables. This part uses a parallel layout $31-33$ to repeat and stress that "adjacency matrices outperform node-link diagrams", a conclusion drawn from the experiment and also emphasized as a corresponding core finding in the original publication. Finally (VI), the comic evaluates the hypotheses and reports which ones are supported and which are unsupported.

Design decisions: The comic makes a set of design decisions to communicate study design and results. As a form of sequential art, comics are naturally averse to organizing hierarchies, so extra care has been taken to ensure clear section structures and semantic nestings. The heading for each part, for example, used large spacing and typography to preserve proper contrast in the masonry layout, aiming for visual clarity and structure $(\mathrm{O} 4)$. To demarcate the canvas regions and bounds of tasks and conditions, the author uses characteristic panels to group each of them $9-10,12-14,15$, and 16. The primary condition "techniques," i.e., node-link diagrams and adjacency matrices, are given different identity colors to be reused 8 (O3). A sketch of the user interface in the experiment is placed in the study setup section to give the audience a clearer understanding of the tasks $22(\mathrm{O} 2)$. The comic also employs a set of data comic design patterns [13], such as flashback 4, space-annotation 20, temporal sequence 6, 25-28, question-andanswer and multiple facets $31-33$.

The visual style (O5) of the comic aims to achieve a balance between clarity (sufficient spacing, reduced and harmonic color palette, consistent visual emphasis, clean, grid-like, and non-overlapping layout, abstraction of concepts), objectivity (few and very small narrators), as well as artistic demand to create a compelling and unique visual experience (hand-drawn style, pictograms).

\section{Stages and Design Solutions}

This section describes the 10 stages which informed the design of our data comics study reports. In our framework, a stage summarizes the information a data comic can show about a specific aspect of the reporting process, and provides visual solutions for common communication needs. A stage can be seen as a short set of guidelines about what information to report in each part of a study and how to represent this information through graphics, text, and a combination thereof. We grouped information into stages based on common reporting structures in HCI and visualization papers, our experience about what information can effectively be shown in context (e.g., tasks, independent variables), and existing guidelines (Section 2.1).

Each stage is illustrated with small comic strips selected from example comics created by the authors of this paper and works co-designed with participants (Section 5). The selected examples demonstrate possible design solutions, and we extract commonly used abstract design patterns from the examples if they exist. For each stage we provide a brief definition, explain the presentation objective and discuss challenges and design considerations. Table 1 summarizes the information presented at each stage and our visual solutions.

\section{Stage \#1-Context and Motivation}

Purpose: This stage aims at a general introduction to the problem studied, the motivation for the study and the research question, and explains any important jargon occurring in the remainder of the study. Depending on the intended audience and their knowledge, additional information about the topic and domain might be necessary. Design: This stage also introduces the visual morphology and terminology for the entire comic, e.g., by explaining concepts visually. For example, Fig. 2 and Fig. 11-4 both introduce basic terms (i.e., 'transition' between 'initial visual state' and 'final visual state'; 'weighted graph') through visual explanations using sequences, arrows, and textual annotations. Keywords are highlighted with color to visually link to corresponding 
Table 1. Overview of the 10 framework stages, including what information is reported (Explains) and the Design Solutions we found.

\begin{tabular}{llll} 
& Stage & Explains & Design Solutions and Examples \\
\hline 1 & Context & $\begin{array}{l}\text { Problem, motivation, jargons \& concepts, } \\
\text { domain, background }\end{array}$ & Explaining domain jargon, sketch context (Fig. 2) \\
\hline 2 & Conditions & Techniques, devices & Condition Symbols (Fig. 3(b) Fig. 3(c)) \\
\hline 3 & Hypotheses & Hypotheses, expected results & Sketch Hypothesis (Fig. 4(b) Fig. 4(a)) \\
\hline 4 & Tasks & Tasks, instructions, answer modalities, collected data & Illustrate Task (Fig. 5(a)) \\
\hline 5 & Stimuli and Materials & Data sets, user interface & Explain the conditions in Stimuli (Fig. 5(b)) \\
\hline 6 & Participants & Power analysis, eligibility, demographics, numbers & Randomization Plot (Fig. 6) \\
\hline 7 & Study Setup & Study environment, steps (training, tasks), study design & Flowchart or timeline (Fig. 4 Fig. 7(b)) \\
\hline 8 & Data Transformations & $\begin{array}{l}\text { Assumption checks (e.g., normality), transformations (e.g., } \\
\text { z-scoring, logarithmic), outlier removal }\end{array}$ & Show Transformation (Fig. 8(b) Fig. 8(c)) Zoom (Fig. 8(c)) \\
\hline 9 & Result Presentation & Explain important messages of a chart, explain error bars & $\begin{array}{l}\text { Difference heatmap (Fig. 9(b), Tips for interpreting the } \\
\text { charts Fig. 9(a)) }\end{array}$ \\
\hline 10 & Hypotheses Evaluation & Which hypotheses are supported by results & $\begin{array}{l}\text { Comparing the sketch from hypotheses with the chart from } \\
\text { result (Fig. 1 34- 36) }\end{array}$ \\
\hline
\end{tabular}

visual explanations, such as 'transition' and the ' - ' in Fig. 2 and ' $a$ change' and ' $\rightarrow$ ' in Fig. 1. The key problems a study addresses can be explicitly stated, as in Fig. 2.

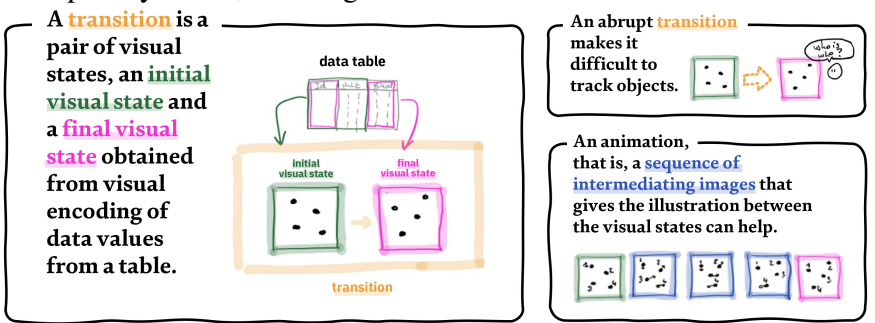

Fig. 2. Example for Stage 1: The comic highlights and explains key concepts ('transition', 'initial visual state', 'final visual state', 'animation') [26] with illustrations of simple examples.

\section{Stage \#2-Conditions}

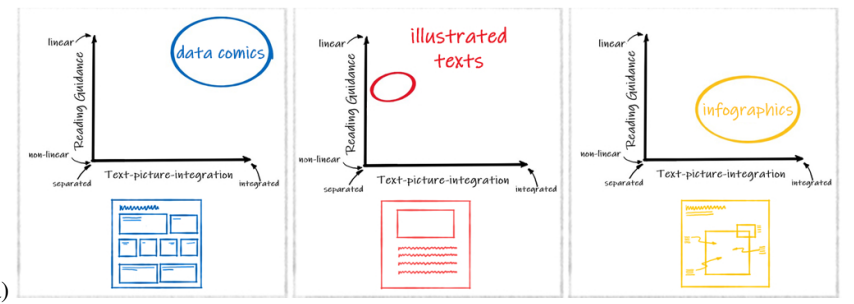

(a)

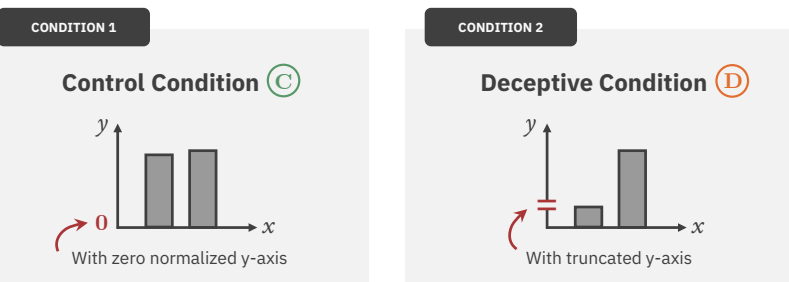

(b)

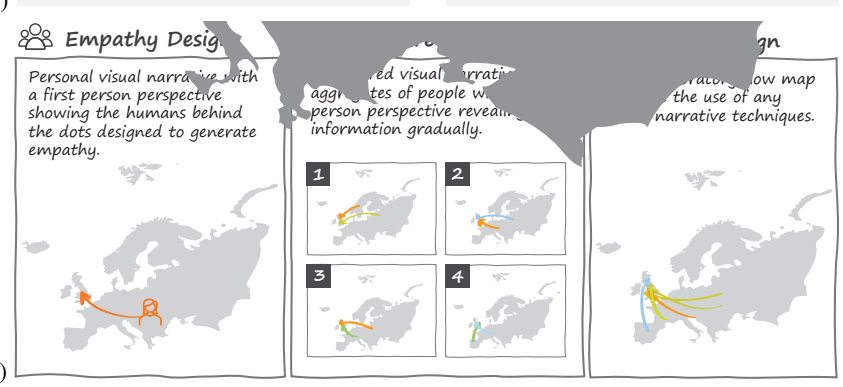

Fig. 3. Examples for Stage 2: Descriptions and iconic condition symbols for (a) visualization types [79] (b) visual encodings [63] (c) two visual narratives compared to a baseline [52].

Purpose: This stage introduces the conditions in a study, i.e., the values chosen for independent study variables. In HCI and visualization this usually includes comparing visualization and interaction techniques, devices, or subsets of a design space. This stage should explain how each condition looks and works, and which ones are the baseline techniques, explaining and illustrating each technique. Design: Conditions can be illustrated through schematic representations (Figures 3(a), 3(b), 3(c)) or, if more iconicity is required, screenshots, photos and other realistic representations. Techniques should be explained well, and for visualization techniques the author should explain how to read each visualization, e.g., through anatomy cheat sheets [78]. Fig. 3(b) shows two tested conditions side-by-side, highlighting the differences in visual encoding (truncated vs. non-truncated y-axis). Fig. 3(a) shows how different techniques relate to a (Cartesian) design space. Symbols can be defined here and re-used to indicate each condition for the remainder of the comic, e.g., using '(C) to present 'control condition' and 'ه8: to present 'Empathy Design'.

\section{Stage \#3-Hypotheses}

Purpose: This stage illustrates and describes the hypotheses tested in the study. A hypothesis can involve one or more conditions, at least one dependent variable, as well as expected outcomes stated in terms of effect size. Design: Illustrating hypotheses can be challenging due to their hypothetical nature. Illustrating the conditions involved can be straightforward, e.g., by repeating figures introduced for conditions (and potentially also for tasks, Fig. 4(b) and Fig. 4(a)). To illustrate hypothetical values for dependent variables, we can use data visualizations with the same encoding as those used to report results, as this makes evaluating hypotheses easier. For a single quantitative dependent variable, we tried bar charts. If a study author pre-registers a hypothesis and a minimum expected effect size, this is straightforward to visualize. However, this is not a well-adopted research practice, and less well-defined hypotheses such as 'larger than', 'no difference' or 'range' are fuzzy and hard to convey in a single bar chart. Annotations could highlight concepts such as differences, averages, values and associated

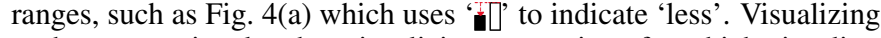
such concepts is related to visualizing uncertainty, for which visualization techniques have been summarized elsewhere [45]. One solution is to omit axis tick marks along axes (Figures 4(b) and 4(a)). Similar presentation is found in research papers [41], for example, Endicott [35] uses schematic diagrams to illustrate hypotheses about the origin of the modern human.

\section{Stage \#4-Tasks and Dependent Variables}

Purpose: This stage illustrates the individual tasks participants perform during the study. This can include instructions given to participants, the way instructions were presented, what participants saw in each task, what they had to do, how they entered an answer to a question and completed a trial, which answers were valid, what data were collected for each dependent variable and how these data were collected. Design: Tasks can be shown through a sequence of actions (Fig. 5(b)), by showing example stimuli, what users needed to pay attention to or count (Fig. 1 12-14) and eventually showing the interaction used to finish a trial and submit an answer (e.g., Fig. 5(b) shows a clicking action, while Fig. 1 (bottom of 12-14) shows a multiple choice menu). 


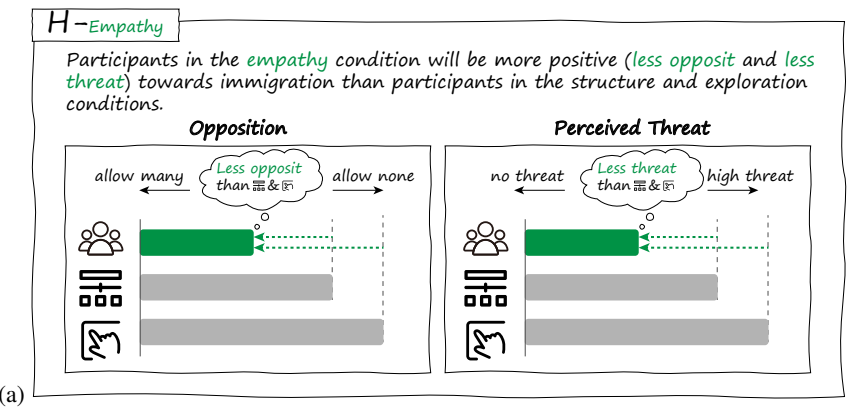

(a)

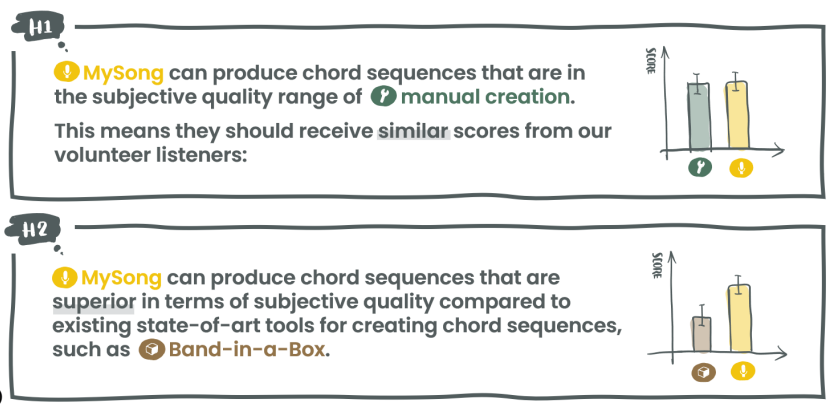

Fig. 4. Examples for Stage 3: Descriptions and sketches of hypotheses for (a) visualization study [52], (b) musical interface evaluation [69].
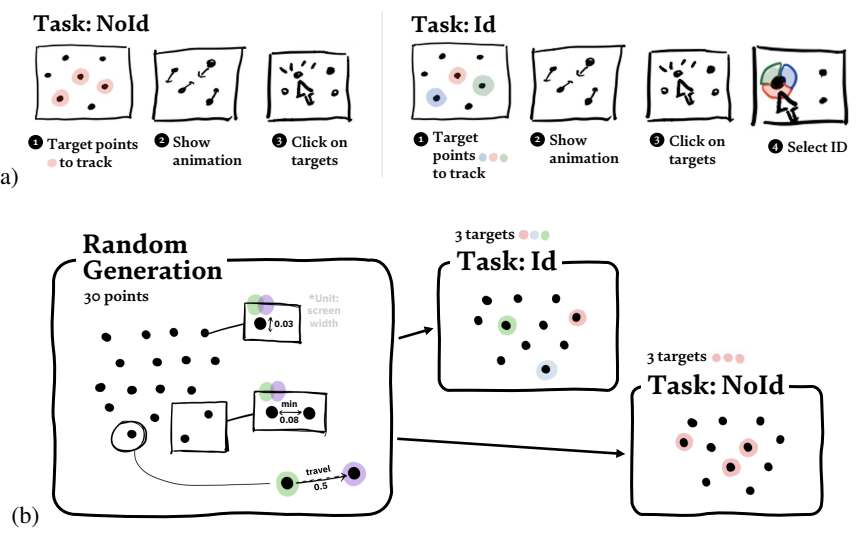

Fig. 5. Example for Stage 4 and Stage 5: (a) how participants in a perceptual study [26] select targets in two conditions (b) how the stimuli are generated for these tasks.

\section{Stage \#5-Stimuli and Materials}

Purpose: This stage should introduce the individual stimuli presented to participants during the study. This is related to the previous stage on Tasks and Dependent Variables but it aims to give examples of stimuli and explains the factors involved in their design. For example, in visualization, this should include the example data sets in the setup participants saw and details like their size and complexity. If data sets are generated, illustrations could show parameters and values. Design: Factors used to generate data sets can be shown by examples, such as Fig. 15- 16 which shows examples of small and large, dense and sparse networks or Fig.5 of [12] showing example stimuli with different parameters (number-of-clusters) as well as the variation of data sets with the same parameters ((c), (d)). The example in Fig. 5(b) shows an example of a data set with randomly generated point positions. Multiple small panels are used to display detailed views of different conditions.

\section{Stage \#6-Participants}

Purpose: This stage should include information about participants themselves (e.g., number, gender balance, eligibility criteria), as well as results of any power analysis used to estimate the number of partici-
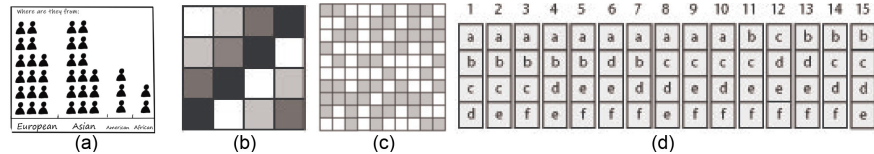

Fig. 6. Examples for Stage 6: (a) participant demographics isotype chart [79], (b) Latin Square randomization, (c) completely randomized design and (d) balanced incomplete block design from [81].

pants required to detect an effect. Design: Fig. 6(a) shows participant demographic data using isotype visualization. A randomization plot can be employed to show various randomization schemes (Fig. 6 (b) Latin Square randomization [81] (c) completely randomized design [2] or (d) balanced incomplete block design [81]) to demonstrate the allocation of participants to stimuli. If power analysis is used to determine sample size, a power curve for the study design [34] can be shown to display the power estimate and its margin of error.

\section{Stage \#7-Study Setup}

Purpose: This stage helps the reader get an overview of the study procedure. This can include the study environment and apparatus, the sequence of steps in a study (e.g., training, trials) as well as detailed information associated with each step (e.g., duration, setup) and the instructions participants were provided with at each step. Design: The sequence of steps can be shown as a pipeline (Fig. 7(b)) or a flowchart (Fig. 7(a)). Annotations provide both an overview of the pipeline and detailed information about participants, materials and duration. Study setup can be complex, and may be explained over several sections in a paper, so the author may be selective about the information reported in this stage.

\section{Stage \#8-Data Transformations and Checks}

Purpose: This stage describes transformations (e.g., log-transform) and checks performed on the data before any significance tests or regression analyses. This stage can include outlier removal and winsorizing, or checking for normality before running statistical tests. Design: Outlier removal can be indicated by thresholds, e.g., at the extreme of a distribution as in Fig. 8(b), and placed alongside descriptions of the nature of the outliers (e.g., as explanations in speech bubbles). Fig. 8(c) shows a distribution before and after transformation; possible visualizations include histograms or $Q-Q$ plots.

\section{Stage \#9-Result Presentation}

Purpose: Compared to the previous stages, this stage may require far greater consideration, given the importance of figures and visualizations for analysis and presentation of results. The information presented can include anything important for understanding the results: distributions, effect sizes, descriptive statistics, error bars or measures of uncertainty. Data visualizations are key in this stage and require appropriate descriptions as to how they should be read [78] as well as annotations to highlight key messages. Design: A number of different techniques may be necessary for this stage: for example, bars in a result bar chart can be reordered to facilitate pair-wise comparison of distributions. Such a reordering can be made explicit through a before-after sequence and, e.g., an arrow highlighting the change (Fig 9(d)). Fig 9(c) uses a cut-out panel to reveal a bimodal distribution with a violin plot. Other statistical results might require showing trends, extreme values, distributions or outliers, which can be facilitated by annotations. In case of complex charts or insights, multiple repetitions of a chart can be presented, each one focusing on a specific message (Fig. 9(b)). To show pair-wise differences between more than two independent variables, Fig. 9(a) uses a "difference heat-map", showing pair-wise differences on different levels $(.01, .001)$.

\section{Stage \#10-Hypothesis Evaluation}

Purpose: This last stage contrasts the initial hypotheses with the findings from the results in \#9. It should show which hypotheses can be accepted and which ones are not supported by the results. Design: Fig. 1, for example, shows both, the figure drawn for the initial hypotheses (\#2) 


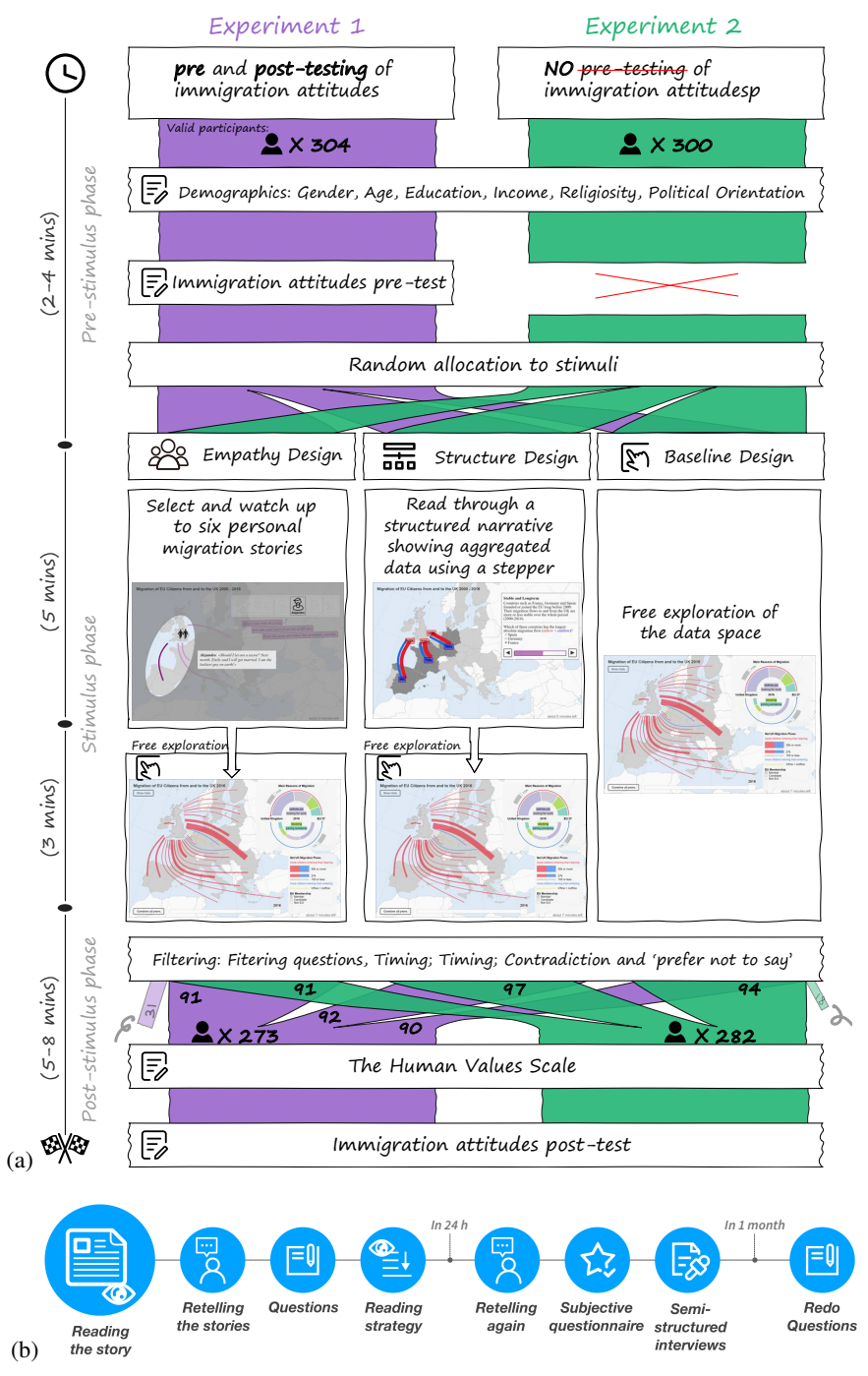

Fig. 7. Examples for Stage 7: (a) pipeline describes the sequence of study steps and how procedures differed between conditions across two experiments [52] (b) A flowchart describes the different phases of a study in chronological order [79].

and the the actual result figure. Supported and unsupported hypotheses can be highlighted with ' $\boldsymbol{Y}$ ' and ' $\boldsymbol{X}$ '.

\section{Collaborative Design Sessions}

We conducted remote collaborative design sessions to evaluate our solutions and stages and obtain feedback, explore more solutions, and to understand which challenges people might encounter in creating data comics for a wider range of studies.

Participants Participants were recruited through email lists at computer science research labs at three universities (two in Europe and one in Canada). We motivated participation by providing guidance on how to create data comics for the students' own studies, co-designed and polished by two of the authors. We gave the comic in Fig. 1 as an example. We did not constrain the scope to controlled studies in HCI and visualization. The seven participants (three females) include five Ph.D. students and two recently graduated master students specialising in usable security and privacy (SecPriv), computational social psychology (CompPsy), geoinformatics (GeoInf), probabilistic machine learning $(M L)$, 3D interactive visualization (3DVis), public health and health system (Health), and systems design engineering (SysEng) respectively. Their respective studies involved a controlled study in data visualization (GeoInf, [52]), an analysis of online open human data. (SecPriv [73]; CompPsy, [25]), observational animal data and model-
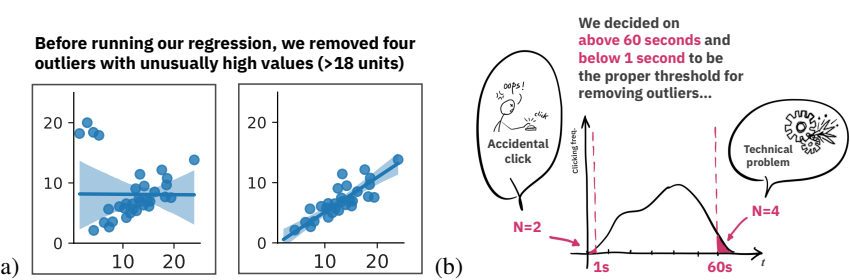

Our data was highly skewed, so we used a logarithmic transformation.

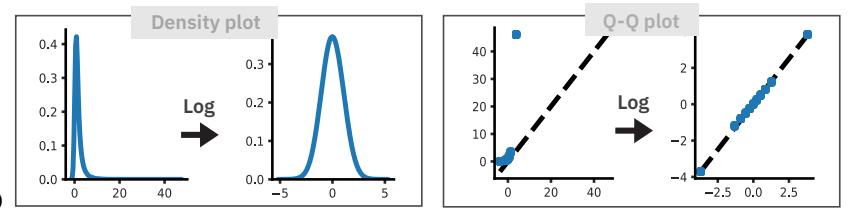

Fig. 8. Examples for Stage 8: (a) Visualizing the process of outlier removal reveals that the goodness of fit in a regression model was highly dependent on outlier removal. (b) Authors can justify criteria for outlier removal using annotated density plots [12] (c) Data comics can demonstrate the effect of important distribution checks and transformations that are often relegated to a textual description.

\begin{tabular}{cl}
\hline 1 & What motivated you in participating in this workshop? \\
\hline 2 & Which issues do you currently see in reporting studies (as a reader/author)? \\
\hline 3 & $\begin{array}{l}\text { Did our stages-framework help? Which challenges did you experience in } \\
\text { doing your story boards / what was the most difficult part, e.g., stages, illus- } \\
\text { trating specific issues? }\end{array}$ \\
\hline 4 & $\begin{array}{l}\text { Creating storyboards, did that change the way you think about reporting } \\
\text { studies? }\end{array}$ \\
\hline 5 & How are you going to use your final comic? \\
\hline 6 & $\begin{array}{l}\text { What did you value about using data comics to reporting studies, what do you } \\
\text { think works about using comics for reporting studies? }\end{array}$ \\
\hline
\end{tabular}

Table 2. Questions asked of the participants in the co-design process.

ing (ML, [20]), 3D interactive system design and evaluation (3DVis, work-in-progress), cognitive behavior study with a game (Health, [24]) and a study of gestural interactions with VR environments (SysEng, work-in-progress). This selection gave us the chance to explore the potential and limits of our design solutions and receive feedback from diverse perspectives. None of the participants had any experience with creating (data) comics.

Procedure The entire design session was conducted individually for each participant, giving participants and facilitators a flexible schedule to work on comics asynchronously offline and communicate about solutions and design iterations.

1. For the first session, participants were asked to attend with a write-up of their study and to give a brief presentation in 5-10 minutes. Three participants presented slides. This gave facilitators and participants an overview and a review of the important stages of the study.

2. Then, we gave a very brief introduction to data comics and showed examples from https://statscomics.github.io/gallery.html.

3. Participants were then asked to create drafts ("storybords") for their comics off-line before the next session. Participants were free to choose drawing techniques, e.g., hand-sketches, vector graphics, photoshop, screenshots, etc). To guide participants and to see if our framework would help them in their process, we provided textual guidelines of each stages (https://statscomics.github.io/guidelines. $\mathrm{html}$ ) as well as the three example comics.

4. After 1-4 days, instructors met again with each participant, discussing storyboards and any challenges encountered.

5. Based on feedback from participants, the instructors iterated upon their comics, aided by slides, written reports and other records.

6. Eventually, participants were asked summative questions (Table 2).

Results Drafts from participants showed that they presented a visual and structured layout of their studies. The structure included our stages, e.g., motivation, methods, conditions, stimulus and results. Design solutions included $a$ ) a Venn diagram to represent the relationship between data and samples (SecPriv, Health); b) a timeline to 


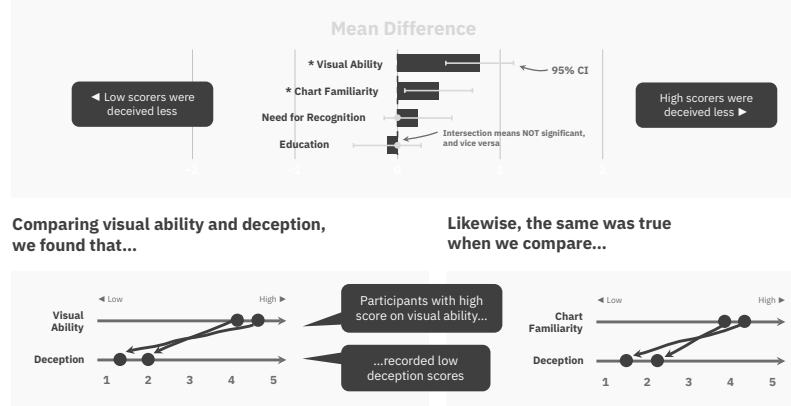

(a)

A Kruskal-Wallis test confirmed a significant difference between conditions for responses to the deception test question, Mann-Whitney $\mathrm{U}$ test with Bonferroni-Halm correction were used as post-hoc tests.
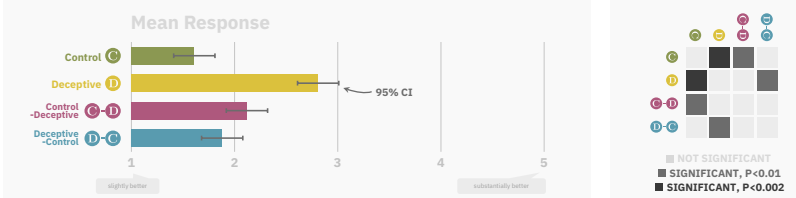

Let's examine each difference in detail between the pairs...
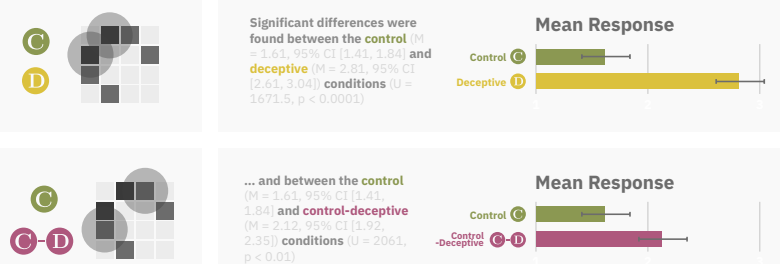

(b)

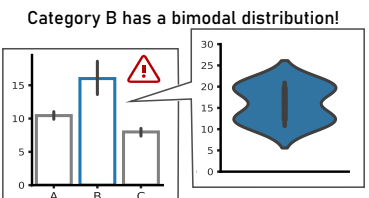

(d)

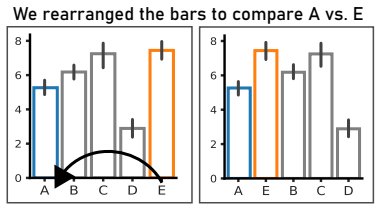

Fig. 9. Examples for Stage 9: (a). A complex result [63] can be presented using a sequence of secondary visualizations (b) or by using multiple explanations of a repeated chart to focus on specific messages. (c) A Zoom pattern [13] can be used to reveal distribution details (d) and panel sequences can illustrate operations like variable re-ordering.

illustrate tasks (GeoInf, CompPsy, 3DVis); c) simple pictographs to demonstrate differences between technical terms (GeoInf, CompPsy, Health, 3DVis, SysEng) (e.g.,'mood' and 'emotion'), and $d$ ) illustration and interpretation of the conditions and materials used in the study (GeoInf, SysEng). All participants presented annotated result charts interpreting features such as significant differences (GeoInf), error bars $(M L)$ and the presence of 'less fluctuation' and 'more fluctuation' in a Gaussian process regression (CompPsy).

Qualitative feedback All participants in the collaborative design session expressed very positive comments about the experience and the results of the session, describing data comics as, e.g., a "visual interface into a more written format" (GeoInf). Participants were motivated to participate in the collaborative design session as it was an "interesting problem" to "expand [the] visibility of [their] research" and to enable "more people get the message" (CompPsy). Participants found data comics a "very good opportunity to [create] a visual, easy to consume format for a blog post" (GeoInf).

About current ways of reporting, participants found the complexity of the information a challenge: "it is often very time consuming to collect all the valuable/required information about a study reported in a classical paper format [...] one has to 'close read' the paper and investigate additional material to find this information, and [expand] the effort to find the right information." (GeoInf). From an author's per- spective, the drawbacks of current reporting include that it is difficult to "convey/communicate the complexity of study designs and/or stimuli in a compelling, easy to understand format." (GeoInf). Moreover, there is little support for grasping the information in a paper: "the current expected paper format/structure does not encourage authors to provide a (standardised) high-level yet detailed summary of such information in a 'front-matter' of a paper (besides the abstract, where detail is limited; or teaser-images)." (GeoInf).

For the eventual use of their comic, participants agreed on general uses of "outreach, publicity" (GeoInf). More concrete scenarios included the use of comics as a "script for [a] conference presentation" $(M L)$ and as suitable material for blog posts, posters ("instead of posters I can also reuse data comics." (SecPriv)), conference presentations and online project pages to "present what we did[,] in a 5 minuteread" (SecPriv). For future, wider use, one participant wished that data comics "would become an accepted format [...] for publishing research" and mentioned potential extensions through "interactive formats like scroll[ing] stories" (GeoInf). This participant also acknowledged the need for guidance and standardization "similar to pre-registration templates or the stages-framework used in the [co-design workshop]."

With respect to the instructional material provided from our side, participants found our motivating examples "well-illustrated" and highlighted that the feedback from instructors was very helpful "since this challenges my perception of other people's understanding” (CompPsy). Our stages helped participants "[c]oming up with an idea of what I want" (SecPriv), to "distill the required information" and create "a structure to follow" (GeoInf). However two participants found it easy to follow the stages since they had already written up their study reports. Stages were also found to be helpful to guide the inclusion or exlusion of information, since they helped participants to focus on the process of textual and visual explanation, as well as "to think about all the other possibilities comics have to communicate a narrative." (GeoInf).

Creating comics involved a set of challenges. Despite positive feedback on our stages, three participants reported issues with deciding what information should be included and identifying the target audience to get "the message across" (CompPsy). This is a common issue in storytelling, which goes beyond the specific domain of visualization. Drawing with pen and paper was reported as an issue by one participant. With respect to individual stages, we found most difficulties arose with stage \#5 (Stimuli and Materials, "explain[ing] multiple datasets/methods"), stage \#7 (Study Setup, “complexity of study designs and the complexity of stimuli") (GeoInf) and stage \#8 (Data Transformations and Checks, "explaining transformations/re-binning of collected information"). Although the stages were given as guidance, one participant found difficulties in deciding "what sequence was the best, the amount of text to include and [the way of] making it easy-tounderstand" (SysEng). A specific challenge arose from the need to explain specific machine learning models $(M L)$, algorithms (CompPsy) and formulas (SysEng). While outside the scope of this paper, this will require significant attention in future work.

Asked whether creating storyboards changed participants' thinking about reporting studies, the answers indicated that participants found "storytelling is important" (Health) and data comics a "very suitable format" (GeoInf) "on top of my list of methods/techniques to report findings or study methods." (SysEng) and would suggest such comics "as a service for researchers" (SecPriv) since they "capture the idea of my study in a very short period of time and the idea stays in their mind" $(M L)$.

\section{Discussion AND FUtURE WORK}

Designing Comics for Studies: Our collaborative design sessions showed that researchers unfamiliar with general visualization practices clearly see the need for better communication and the potential of techniques such as data comics. Moreover, our stages discussed in Sect. 4, alongside examples, helped participants understand this novel medium and guide them in expressing their own ideas visually as storyboards. As crafting sophisticated visual content for was found to be an intimidating task by some participants, our collaborative sessions employed a 2-step iterative co-design process, comprising of clear in- 
structions and guidelines about what was expected from a participant (stages, storyboard) and the involvement of a professional designer polishing the storyboard and helping with design solutions. We believe this process can be refined for future, including more extensive workshops for illustrating studies and for data comics in general [77]. Further workshops and teaching practice will help refine our methodologies and build a formal set of guidelines, instructions, and design templates. An open question is to what extent we can guide decisions on level-of-detail and better design data comics for specific audiences.

Design Objectives: Our collection of comics represents a series of points in the solution space for visually explaining procedures and findings in comic-style study reports. We use this section to reflect on how these relate to our initial design objectives (O1-O5).

Our first design goals were aimed at finding ways to visually explain (O2) key concepts (O1). These concepts were represented through different types of graphics; for example, iconic illustrations were used to display contextual messages related to the goals of the study. Schematics were used to present study methodologies or task workflows. Screenshots were used to present realistic representations of prototype UIs and interactions performed by study participants. Where iconic representations where not possible, e.g., due to the visual space required (e.g., graph sizes in Fig. 1 15-16) or the abstract nature of the concept (e.g., a technique) the designer used more symbolic representations to create a visual vocabulary (03). Data visualizations were naturally used to present results, but also to show demographics and data transformations. Some figures explain visualizations and charts, e.g., error-bars, chart axes, or user interface components (GeoInf).

We found some solutions were able to serve as patterns or templates, similar to data comics design patterns [13], supporting transparency and understanding in visual explanations. Examples include step-by-step explanations of tasks (e,g, Fig. 5(a)) making evidence for key claims visually apparent, e.g., facilitating comparison by reordering the bars in a bar chart (Fig. 9(d)); and evaluating hypotheses by comparing schematic visualizations of hypotheses with the actual results (Fig. 1).

Our criteria of visual overview (O4) refers to the ability to provide information at different levels of detail through overview and details. For example, by structuring a comic layout into larger and smaller panels, headlines and subsections, and nested subpanels, and placing panels in clear sequence (Fig. 125-28) to show a temporal relation, or in parallel (Fig. 131-33) to show information of parallel meaning. For complex layouts, arrows indicate reading direction and avoid "backlocks" [28].

We seek visual consistency in illustration style; colors used for highlighting and indicating different conditions; and visual lexicons for individual objects or actions in a comic $(\mathbf{O 3})$. This visual vocabulary, which is gradually introduced to the audience in the early stages of the narrative framework, allows readers to build associations between different experimental concepts and to quickly refer back.

It should be noted that using conventional comic-style presentation does not indicate poor quality $(\mathbf{O 5})$. This aesthetic is chosen with the goal of complementing the textual annotations, and reducing visuals that are irrelevant to a viewer's understanding of the study report. While the style of data comics was not focus of our work, we believe there are many open research questions about the impact of the visual style for understanding, as well as on attractiveness and credibility [80].

Limitations and Open Questions: The research presented in this paper is clearly limited by the number of examples we could produce and the number of interactions we could have with the participants in our design discussion and collaborative design sessions. Unlike for infographics [19,27,54], data videos [9], or data comics [14], there is no corpus that we could curate and learn from to understand best practices, or the effectiveness of existing data comics for reporting empirical studies. Our research has created an initial set of such examples, developed through design exploration and iterative collaborative design, that we make available on our website: https://statscomics.github.io. Our results are only the beginning of a much larger exploration of this vast design space that is yet to be structured and formally evaluated through, e.g., controlled user studies to assess which of our solutions work best and to investigate the effect of various factors on effective scientific communication for various audiences with respect to comprehension, memorability, engagement, and educational value.

Providing better support for authoring data comics is another item to be added to the research agenda. Creating comics involves many skills [77] and authoring support for specialized comics such as data comics [50] is still in its infancy. Possibly, authoring tools could be integrated with data exploration and analysis platforms such as Python notebooks. One challenge with creating such tools is to find the right balance between expressive power and usability, as well as between guidance and creative freedom [67]. This is further complicated by the wide range of elements and concepts in study reports, and the rich visual vocabulary afforded by data comics.

More detailed and specialized guidelines for common study protocols (e.g., Fitt's Law studies) could also help address challenges raised by the participants, some of whom found complex study designs difficult to communicate. Guidelines could also be refined according to a particular comic's intended use case, by indicating to authors the appropriate level of simplification for their audience. Without additional guidance, there is the risk that authors might leave out important details when attempting to communicate to a non-technical audience.

We have deliberately left out in our work the explanation of statistical and other tests, algorithms, and complex systems (though this was mentioned as a difficult point in the process by several participants). For example, while descriptive statistics and regression analysis are commonly explained using graphics, more sophisticated methods pose challenges to understanding and transparency, and could benefit from research into how they could be communicated using comics.

Towards better support for reporting studies. During our research, we engaged in co-design with researchers from economics, cyber-security, computational social psychology, machine learning, geo-informatics, 3D scientific visualization, and HCI. In this paper we provide design solutions of what one could do. While some fields rely on data analysis and modelling uncommon in HCI, others conduct mostly qualitative research, where data comprises of interview records and other observations. We do not yet provide specific visual solutions for such scenarios, but these examples can inform future research.

Moreover, the boundaries between comics for reporting studies and engaging in public outreach begin to blur, e.g., $\mathrm{PhD}$ Comics [22] and xkcd [57] are examples of science-related comics that often "go viral" [23]. We believe that, as comics are a universal communication medium deeply rooted in human culture, data comics (and their close cousins, infographics and graphical abstracts) will receive greater consideration in the near future, helping to narrow the gaps between scientists and the general public, and between communicators and audiences. These formats can inform the design of better graphics in papers, appendices, webpages, talks and slideshows, posters, and videos. Actively engaging in making visual information more commonplace can be transformative to communication: as scientists begin to think about their research more visually, the media for communicating and transmitting thoughts will evolve, and the audience will in turn become more visually literate. We encourage researchers and science communicators to seek inspiration in our work to go beyond the preconceptions associated with comics: data comics and sketching are less about cartoons or particular visual styles and more about thinking, logic, and effective communication. We hope our work will contribute to a new culture of reporting and discussing scientific findings, and to a discussion of transparency, teaching statistics, and data literacy in general.

\section{Conclusion}

This paper explores data comics for illustrating reports of controlled studies. Though an iterative co-design process, we defined common design solutions, guided by five design objectives. Our results and co-design experience are encouraging, and can inform further research on improving scientific reporting. We contribute a set of examples of how comics support that reporting, providing an initial set of concrete ideas for how to communicate common stages of HCI studies and statistical analysis, and may serve as a starting point for future exploration. Finally, we show that our new method allows people to create clear and compelling visual summaries of complex research designs. 


\section{REFERENCES}

[1] Distill.pub. https://distill.pub/, 2020

[2] Experimental design and analysis of variance. //yieldingresults.org/modules/module-2/, 2020.

[3] medium.com. https://medium.com/, 2020.

[4] Publication Manual of the American Psychological Association, Seventh Edition. American Psychological Association, 2020.

[5] Researchblogging.org. http://researchblogging.org/, 2020

[6] Seeing theory. https://seeing-theory.brown. edu/, 2020.

[7] B. Alper, B. Bach, N. Henry Riche, T. Isenberg, and J.-D. Fekete. Weighted graph comparison techniques for brain connectivity analysis. In Proceedings of the 2013 CHI Conference on Human Factors in Computing Systems, pp. 483-492. ACM, 2013.

[8] S. V. Amaral, T. Forte, J. Ramalho-Santos, and M. T. G. da Cruz. I want more and better cells!-an outreach project about stem cells and its impact on the general population. PloS one, 10(7), 2015.

[9] F. Amini, N. Henry Riche, B. Lee, C. Hurter, and P. Irani. Understanding data videos: Looking at narrative visualization through the cinematography lens. In Proceedings of the 33rd Annual ACM Conference on Human Factors in Computing Systems, pp. 1459-1468, 2015.

[10] B. Bach, N. Kerracher, K. W. Hall, S. Carpendale, J. Kennedy, and N. Henry Riche. Telling stories about dynamic networks with graph comics. In Proceedings of the 2016 CHI Conference on Human Factors in Computing Systems, pp. 3670-3682. ACM, 2016.

[11] B. Bach, N. H. Riche, S. Carpendale, and H. Pfister. The emerging genre of data comics. IEEE computer graphics and applications, 37(3):6-13, 2017.

[12] B. Bach, R. Sicat, J. Beyer, M. Cordeil, and H. Pfister. The hologram in my hand: How effective is interactive exploration of $3 \mathrm{~d}$ visualizations in immersive tangible augmented reality? IEEE transactions on visualization and computer graphics, 24(1):457-467, 2017.

[13] B. Bach, Z. Wang, M. Farinella, D. Murray-Rust, and N. Henry Riche. Design patterns for data comics. In Proceedings of the 2018 CHI Conference on Human Factors in Computing Systems, p. 1-12. ACM, New York, NY, USA, 2018. doi: 10.1145/3173574.3173612

[14] B. Bach, Z. Wang, N. Henry Riche, M. Farinella, D. Murray-Rust, S. Carpendale, and H. Pfister. Data comics. https://www . datacomics. net/, 2017-2020.

[15] S. K. Badam, Z. Liu, and N. Elmqvist. Elastic documents: Coupling text and tables through contextual visualizations for enhanced document reading. IEEE transactions on visualization and computer graphics, 2019.

[16] A. Barberousse. What is the use of diagrams in theoretical modeling? Science in Context, 26(2):345-362, 2013.

[17] C. B. Begg. Publication bias. The handbook of research synthesis, 25:299409, 1994.

[18] R. Beyth-Marom, F. Fidler, and G. Cumming. Statistical cognition: Towards evidence-base practice in statistics and statistics education. Statistics Education Research Journal, 7(2), 2008.

[19] M. A. Borkin, A. A. Vo, Z. Bylinskii, P. Isola, S. Sunkavalli, A. Oliva, and $\mathrm{H}$. Pfister. What makes a visualization memorable? IEEE transactions on visualization and computer graphics, 19(12):2306-2315, 2013.

[20] M. P. Camilleri and C. K. Williams. The extended dawid-skene model: Fusing information from multiple data schemas. arXiv preprint arXiv: 1906.01251, 2019.

[21] I. Chalmers and P. Glasziou. Avoidable waste in the production and reporting of research evidence. The Lancet, 374(9683):86-89, 2009.

[22] J. Cham. Phd comics. http://phdcomics.com/.

[23] J. Cham. The science gap. https://tedx.ucla.edu/talks/jorge_ cham_the_science_gap/, 2012.

[24] Chan, Long Ting. Leveraging the proteus effect to motivate emotional support in a serious game for mental health, 2019.

[25] L. L. Chen, W. Magdy, H. Whalley, and M. Wolters. Examining the role of mood patterns in predicting self-reported depressive symptoms. 2020.

[26] F. Chevalier, P. Dragicevic, and S. Franconeri. The not-so-staggering effect of staggered animated transitions on visual tracking. IEEE transactions on visualization and computer graphics, 20(12):2241-2250, 2014.

[27] F. Chevalier, R. Vuillemot, and G. Gali. Using concrete scales: A practical framework for effective visual depiction of complex measures. IEEE transactions on visualization and computer graphics, 19(12):2426-2435, 2013.

[28] N. Cohn. Navigating comics: an empirical and theoretical approach to strategies of reading comic page layouts. Frontiers in psychology, 4:186,
2013.

[29] N. Cohn. The Visual Language of Comics: Introduction to the Structure and Cognition of Sequential Images. A\&C Black, 2013.

0] M. Correll and M. Gleicher. Error bars considered harmful: Exploring alternate encodings for mean and error. IEEE transactions on visualization and computer graphics, 20(12):2142-2151, 2014.

[31] N. Desrochers, A. Paul-Hus, S. Haustein, R. Costas, P. Mongeon, A. QuanHaase, T. D. Bowman, J. Pecoskie, A. Tsou, and V. Larivière. Authorship, citations, acknowledgments and visibility in social media: Symbolic capital in the multifaceted reward system of science. Social science information, 57(2):223-248, 2018.

[32] P. Dragicevic. Fair statistical communication in hci. In Modern Statistical Methods for HCI, pp. 291-330. Springer, 2016.

[33] P. Dragicevic, Y. Jansen, A. Sarma, M. Kay, and F. Chevalier. Increasing the transparency of research papers with explorable multiverse analyses. In Proceedings of the 2019 CHI Conference on Human Factors in Computing Systems, pp. 1-15. ACM, 2019.

[34] A. Eiselmayer, C. Wacharamanotham, M. Beaudouin-Lafon, and W. E. Mackay. Touchstone2: An interactive environment for exploring trade-offs in hci experiment design. In Proceedings of the 2019 CHI Conference on Human Factors in Computing Systems, pp. 217:1-217:11. ACM, New York, NY, USA, 2019. doi: 10.1145/3290605.3300447

[35] P. Endicott, S. Y. Ho, and C. Stringer. Using genetic evidence to evaluate four palaeoanthropological hypotheses for the timing of neanderthal and modern human origins. Journal of human evolution, 59(1):87-95, 2010.

[36] M. Farinella. The potential of comics in science communication. Journal of science communication, 17(01):Y01-1, 2018.

[37] L. Gonick and W. Smith. The cartoon guide to statistics. Collins, 1993.

[38] D. Graeber. The utopia of rules: On technology, stupidity, and the secret joys of bureaucracy. Melville House, 2015.

[39] T. Grossman, F. Chevalier, and R. H. Kazi. Your paper is dead! bringing life to research articles with animated figures. In Proceedings of the 33rd Annual ACM Conference Extended Abstracts on Human Factors in Computing Systems, pp. 461-475, 2015.

[40] C. Group. Consort: Consolidated standards of reporting trials. http: //www. consort-statement.org/, 2010

[41] W.-C. Ho, Y. Ohya, and J. Zhang. Testing the neutral hypothesis of phenotypic evolution. Proceedings of the National Academy of Sciences, 114(46):12219-12224, 2017.

[42] K. Hornbæk, S. S. Sander, J. A. Bargas-Avila, and J. Grue Simonsen. Is once enough? on the extent and content of replications in human-computer interaction. In Proceedings of the 2014 CHI Conference on Human Factors in Computing Systems, pp. 3523-3532. ACM, 2014.

[43] J. Hosler and K. Boomer. Are comic books an effective way to engage nonmajors in learning and appreciating science? CBE-Life Sciences Education, 10(3):309-317, 2011.

[44] J. Hullman and B. Bach. Picturing science: Design patterns in graphical abstracts. In International Conference on Theory and Application of Diagrams, pp. 183-200. Springer, 2018.

[45] J. Hullman, X. Qiao, M. Correll, A. Kale, and M. Kay. In pursuit of error: A survey of uncertainty visualization evaluation. IEEE transactions on visualization and computer graphics, 25(1):903-913, 2018.

[46] S. Huron, S. Carpendale, J. Boy, and J.-D. Fekete. Using viskit: A manual for running a constructive visualization workshop. 2016.

[47] M. Kang. Measuring social media credibility: A study on a measure of blog credibility. Institute for Public Relations, pp. 59-68, 2010.

[48] K. Karaca. Representing experimental procedures through diagrams at cern's large hadron collider: The communicatory value of diagrammatic representations in collaborative research. Perspectives on science, 25(2):177-203, 2017

[49] C. Kilkenny, W. J. Browne, I. C. Cuthill, M. Emerson, and D. G. Altman. Improving bioscience research reporting: the arrive guidelines for reporting animal research. PLoS biology, 8(6), 2010

[50] N. W. Kim, N. Henry Riche, B. Bach, G. Xu, M. Brehmer, K. Hinckley, M. Pahud, H. Xia, M. J. McGuffin, and H. Pfister. Datatoon: Drawing dynamic network comics with pen+ touch interaction. In Proceedings of the 2019 CHI Conference on Human Factors in Computing Systems, pp. $1-12$. ACM, 2019.

[51] R. B. Kline. What's wrong with statistical tests-and where we go from here. 2004.

[52] J. Liem, C. Perin, and J. Wood. Structure and empathy in visual data storytelling: Evaluating their influence on attitude. In Computer Graphics Forum. Wiley Online Library, 2020. 
[53] S.-F. Lin, H.-s. Lin, L. Lee, and L. D. Yore. Are science comics a good medium for science communication? the case for public learning of nanotechnology. International Journal of Science Education, Part B, 5(3):276-294, 2015.

[54] M. Lu, C. Wang, J. Lanir, N. Zhao, H. Pfister, D. Cohen-Or, and H. Huang. Exploring visual information flows in infographics. In Proceedings of the 2020 CHI Conference on Human Factors in Computing Systems, pp. 1-12. ACM, 2020.

[55] M. Lynch and S. Woolgar. Representation in scientific practice. 1990.

[56] S. McCloud. Understanding comics: The invisible art. Northampton, Mass, 1993.

[57] R. Munroe. Xkcd. https://xkcd.com/.

[58] A. Negrete and C. Lartigue. Learning from education to communicate science as a good story. Endeavour, 28(3):120-124, 2004.

[59] M. O'Donnell. Why doctors don't read research papers: scientific papers are not written to disseminate information. BMJ: British Medical Journal, 330(7485):256, 2005.

[60] C. Olah and S. Carter. Research debt. Distill, 2(3):e5, 2017.

[61] L. Pauwels. Visual cultures of science: rethinking representational practices in knowledge building and science communication. UPNE, 2006.

[62] R. Peck, C. Olsen, and J. L. Devore. Introduction to statistics and data analysis. Nelson Education, 2011.

[63] J. Ritchie, D. Wigdor, and F. Chevalier. A lie reveals the truth: Quasimodes for task-aligned data presentation. In Proceedings of the $2019 \mathrm{CHI}$ Conference on Human Factors in Computing Systems, pp. 1-13. ACM, 2019.

[64] H. R. Rothstein, A. J. Sutton, and M. Borenstein. Publication bias in meta-analysis. Publication bias in meta-analysis: Prevention, assessment and adjustments, pp. 1-7, 2005.

[65] S. Ruan, J. O. Wobbrock, K. Liou, A. Ng, and J. A. Landay. Comparing speech and keyboard text entry for short messages in two languages on touchscreen phones. Proceedings of the ACM on Interactive, Mobile, Wearable and Ubiquitous Technologies, 1(4):1-23, 2018.

[66] J. D. Scargle. Publication bias (the" file-drawer problem") in scientific inference. arXiv preprint physics/9909033, 1999.

[67] B. Shneiderman. Creativity support tools: Accelerating discovery and innovation. Communications of the ACM, 50(12):20-32, 2007.

[68] I. Simera, D. Moher, J. Hoey, K. F. Schulz, and D. G. Altman. A catalogue of reporting guidelines for health research. European journal of clinical investigation, 40(1):35-53, 2010.

[69] I. Simon, D. Morris, and S. Basu. Mysong: automatic accompaniment generation for vocal melodies. In Proceedings of the SIGCHI conference on human factors in computing systems, pp. 725-734. ACM, 2008.

[70] A. N. Spiegel, J. McQuillan, P. Halpin, C. Matuk, and J. Diamond. Engaging teenagers with science through comics. Research in science education, 43(6):2309-2326, 2013.

[71] H. Strobelt, D. Oelke, C. Rohrdantz, A. Stoffel, D. A. Keim, and O. Deussen. Document cards: A top trumps visualization for documents. IEEE transactions on visualization and computer graphics, 15(6):11451152, 2009.

[72] D. F. Stroup, J. A. Berlin, S. C. Morton, I. Olkin, G. D. Williamson, D. Rennie, D. Moher, B. J. Becker, T. A. Sipe, S. B. Thacker, et al. Metaanalysis of observational studies in epidemiology: a proposal for reporting. Jama, 283(15):2008-2012, 2000.

[73] M. Tahaei, K. Vaniea, and N. Saphra. Understanding privacy-related questions on stack overflow. 2020.

[74] S. Takahashi et al. The manga guide to statistics. No Starch Press, 2008.

[75] B. Victor. Explorable explanations. Online. http://worrydream.com/ ExplorableExplanations/, 2011.

[76] B. Victor. Scientific communication as sequential art. Online. http:// worrydream.com/ScientificCommunicationAsSequentialArt/, 2011.

[77] Z. Wang, H. Dingwall, and B. Bach. Teaching data visualization and storytelling with data comic workshops. In Extended Abstracts of the 2019 CHI Conference on Human Factors in Computing Systems, pp. 1-9. ACM, 2019.

[78] Z. Wang, L. Sundin, D. Murray-Rust, and B. Bach. Cheat sheets for data visualization techniques. In Proceedings of the 2020 CHI Conference on Human Factors in Computing Systems, p. 1-13. ACM, New York, NY, USA, 2020. doi: 10.1145/3313831.3376271

[79] Z. Wang, S. Wang, M. Farinella, D. Murray-Rust, N. Henry Riche, and B. Bach. Comparing effectiveness and engagement of data comics and infographics. In Proceedings of the 2019 CHI Conference on Human
Factors in Computing Systems, pp. 1-12, 2019.

[80] J. Wood, P. Isenberg, T. Isenberg, J. Dykes, N. Boukhelifa, and A. Slingsby. Sketchy rendering for information visualization. IEEE transactions on visualization and computer graphics, 18(12):2749-2758, 2012.

[81] S. C. Yarnes. Introduction to randomization and layout. Online. https: //pbgworks.org/index.php?q=node/1534, 2013.

[82] P. T. Zellweger, B.-W. Chang, and J. D. Mackinlay. Fluid links for informed and incremental link transitions. In Proceedings of the ninth ACM conference on Hypertext and hypermedia: links, objects, time and spacestructure in hypermedia systems: links, objects, time and space-structure in hypermedia systems, pp. 50-57. ACM, 1998.

[83] P. T. Zellweger, A. Mangen, and P. Newman. Reading and writing fluid hypertext narratives. In Proceedings of the thirteenth ACM conference on Hypertext and hypermedia, pp. 45-54. ACM, 2002.

[84] Z. Zhao, R. Marr, and N. Elmqvist. Data comics: Sequential art for data-driven storytelling. tech. report, 2015. 